MARIANGELA BADAIOTTI

AdRIANA ARENT ${ }^{2}$

VICENTE MONTEGGIA ${ }^{3}$

JULIA MACHADO ${ }^{4}$

Rafaella Petracco 5

Alvaro Petracco 6

Relato de Caso

Palavras-chaves

Útero/anormalidades Anormalidades urogenitais Colo do útero/anormalidades

Anormalidades múltiplas Gestação

Ultra-sonografia

Keywords

Uterus/abnormalities Urogenital abnormalities Cervix uteri/abnormalities Abnormalities, multiple Pregnancy

Ultrasonography

Correspondência:

Adriana Arent Rua Quintino Bocaiúva, 1.617/301 CEP 90440-051 - Porto Alegre/RS Fone: (51) 3339-1142 Fax: (51) 3339-1142 E-mail: adriarent@ig.com.br ou fertilitat@fertilitat.com.br

Recebido

07/04/2006

Aceito com modificaçōes $30 / 05 / 2007$

\section{Septo uterino, duplicação cervical e septo vaginal: relato de rara malformação mülleriana com gestação a termo}

\author{
Septate uterus, cervical duplication and vaginal septum: \\ a report of an uncommon malformation with normal term pregnancy
}

\section{Resumo}

Apresentamos um caso de gestação espontânea em uma paciente com útero septado completo e duplicação cervical. Paciente com 34 anos, branca, nuligesta, ciclos regulares, com suspeita de septo uterino em exame ecográfico. Ao exame, apresentava septo vaginal longitudinal até a região himenal e dois colos uterinos. Solicitada ecografia pélvica tridimensional que evidenciou duplicação cervical, septo uterino do istmo à cavidade endometrial e ausência de divisão do corpo uterino, compatível com útero septado completo e duplicação cervical verdadeira. Um mês após, relatou relação sexual desprotegida e atraso menstrual. Ao exame ecográfico foi visualizado saco gestacional único na cavidade uterina direita. Apresentou gestação sem intercorrências. A cesariana ocorreu com 37 semanas, com recém-nascido do sexo feminino saudável e puerpério normal. Esse caso ilustra uma gestação espontânea, sem intercorrências, em uma rara anomalia, cujo impacto reprodutivo ainda não está totalmente elucidado.

\section{Abstract}

This report describes an unusual case of spontaneous pregnancy in a patient with Müllerian anomaly. The patient was a 34-years old, white, nulligravida, with regular menstrual cycles, and suspected uterine septum observed during a routine ultrasonographic examination. The gynecological examination revealed a complete longitudinal vaginal septum and two uterine cervices. Three-dimensional pelvic ultrasonography showed cervix duplication, uterine septum from isthmus to endometrial cavity and absence of uterine body division, compatible with complete uterine septum and true dual cervices. She returned after one month and reported unprotected sexual intercourse and delayed menstrual period. She was pregnant, had a good pregnancy evolution, and delivered a healthy term baby girl, by cesarean section, at 37 weeks of pregnancy. This report describes a case of normal-term pregnancy in a patient with a rare anomaly (vaginal septum and two cervices) who became spontaneously pregnant without treatment.

\section{Trabalho realizado no Fertilitat/Centro de Medicina Reprodutiva - Porto Alegre (RS), Brasil.}

' Professora da Faculdade de Medicina da Pontifícia Universidade Católica do Rio Grande do Sul - PUC-RS - Rio Grande do Sul (RS), Brasil; Chefe do Serviço de Ginecologia do Hospital São Lucas da Pontifícia Universidade Católica do Rio Grande do Sul - HSL/ PUC-RS - Rio Grande do Sul (RS), Brasil; Diretora do Fertilitat/Centro de Medicina Reprodutiva - Rio Grande do Sul (RS), Brasil. 2 Preceptora do Serviço de Ginecologia do Hospital São Lucas da Pontifícia Universidade Católica do Rio Grande do Sul - HSL/PUC-RS - Rio Grande do Sul (RS), Brasil; Membro do Fertilitat/Centro de Medicina Reprodutiva - Rio Grande do Sul (RS), Brasil. ${ }^{3}$ Médico Ginecologista do Serviço de Ginecologia e Obstetrícia do Hospital São Lucas.

${ }_{4}^{4}$ Residente do Serviço de Ginecologia e Obstetrícia do Hospital São Lucas da Pontifícia Universidade Católica do Rio Grande do Sul - HSL/PUC-RS - Rio Grande do Sul (RS), Brasil.

${ }^{5}$ Residente do Serviço do Complexo Hospitalar Santa Casa de Porto Alegre - Rio Grande do Sul (RS), Brasil.

- Professor da Faculdade de Medicina da Pontifícia Universidade Católica do Rio Grande do Sul - PUC-RS - Rio Grande do Sul (RS), Brasil; Diretor do Fertilitat/Centro de Medicina Reprodutiva - Rio Grande do Sul (RS), Brasil.

Conflito de interesses: Os autores deste estudo não apresentam nenhum interesse financeiro ou de qualquer outra natureza relacionados à publicação deste artigo, cujo cunho é somente científico. 


\section{Introdução}

No desenvolvimento mülleriano normal, parece existir uma progressão ordenada do desenvolvimento embrionário do útero, tubas uterinas e vagina. Alterações deste processo resultam em anomalias uterinas, tubárias e/ou vaginais congênitas. As malformações müllerianas representam um conjunto de anormalidades estruturais que podem se originar da fusão destes dutos na linha média na falha na conexão com o seio urogenital, ou na falha quanto a criar uma luz apropriada na porção superior da vagina e útero, por não reabsorção das células da vagina central e do septo entre os dutos müllerianos fundidos ${ }^{1}$. Porém, a causa definitiva destas anomalias não está claramente elucidada. Estima-se que acometam, aproximadamente, 1 a 6\% das mulheres, porém a incidência exata destas malformações é incerta, pois a maioria dos dados provém de estudos de complicações obstétricas ou de infertilidade ${ }^{1,2}$. Várias classificações foram propostas para as anomalias uterinas congênitas, baseadas no entendimento da embriologia do sistema mülleriano, sendo a mais utilizada a posposta por Buttram e Gibbons ${ }^{3}$, posteriormente modificada pela American Fertility Society ${ }^{4}$.

Dentre as anormalidades congênitas, o septo uterino está associado com maior incidência de falha reprodutiva e complicações obstétricas, incluindo aborto recorrente de primeiro e segundo trimestre, parto prematuro, anormalidades da apresentação fetal, retardo de crescimento intra-uterino e infertilidade. Aproximadamente 15 a $25 \%$ dos abortos espontâneos são causados por defeitos da fusão dos ductos müllerianos, sendo grande parte associados a septo uterino 5 .

O septo uterino é a malformação uterina congênita mais comum, porém apenas $14 \%$ das pacientes com septo uterino apresentam um septo completo com envolvimento cervical. Ainda mais rara é a ocorrência de duplicação cervical verdadeira associada a septo uterino completo e septo vaginal longitudinal. Esta é uma malformação que, além de rara, não se enquadra na teoria clássica de desenvolvimento embrionário e na classificação da ASRM, estando descritos menos de 40 casos na literatura ${ }^{6,7}$.

Esta anomalia já foi descrita previamente pelos autores $^{8}$, mas apresentamos agora o relato de gestação espontânea nesta paciente, que evoluiu a termo, no intuito de alertar a possibilidade deste tipo de malformação, bem como avaliar o seu impacto reprodutivo.

\section{Relato do caso}

Paciente de 34 anos, branca, nuligesta, veio à consulta por suspeita de septo uterino em exame ecográfico bidimensional. Referia desenvolvimento puberal normal com menarca aos 12 anos, ciclos menstruais regulares, com quatro dias de fluxo normal e dismenorréia leve. Negava dispareunia. Não apresentava história materna de exposição a dietilbestrol e não havia conhecimento de malformações de sistema urinário.

Ao exame ginecológico, apresentava genitália externa normal e septo vaginal longitudinal, que se estendia até à região himenal, e presença de dois colos uterinos. No toque vaginal palpavam-se os dois colos, o útero era anteversofletido de volume usual e não havia tumores pélvicos. A ecografia transvaginal demonstrava duas cavidades uterinas com distância intercornual normal e alargamento da região cervical. Para elucidação diagnóstica, foi solicitada ecografia pélvica tridimensional, que evidenciou duplicação cervical (Figura 1), septo uterino que se estendia do istmo ao final da cavidade endometrial e ausência de divisão do corpo uterino, compatível com útero septado completo e duplicação cervical verdadeira.

A paciente retornou à consulta um mês depois, relatando relação sexual desprotegida e atraso menstrual. Ao exame ecográfico, foi visualizado saco gestacional único na cavidade uterina direita. A gestação evoluiu sem intercorrências. O parto ocorreu com 37 semanas, por cesariana, durante a qual foi comprovada a ausência de divisão uterina. O recém-nascido do sexo feminino apresentou peso adequado para a idade gestacional, ausência de malformações e Apgar 9/10. A evolução puerperal foi normal.

$\mathrm{O}$ anonimato da paciente foi preservado neste relato, sendo apresentados apenas dados da história médica, sem identificação da mesma. A paciente consentiu em disponibilizar a utilização de seus dados para fins científicos, sendo esse procedimento aprovado pelo Comitê de Ética e Pesquisa da Pontifícia Universidade Católica do Rio Grande do Sul (PUC-RS).

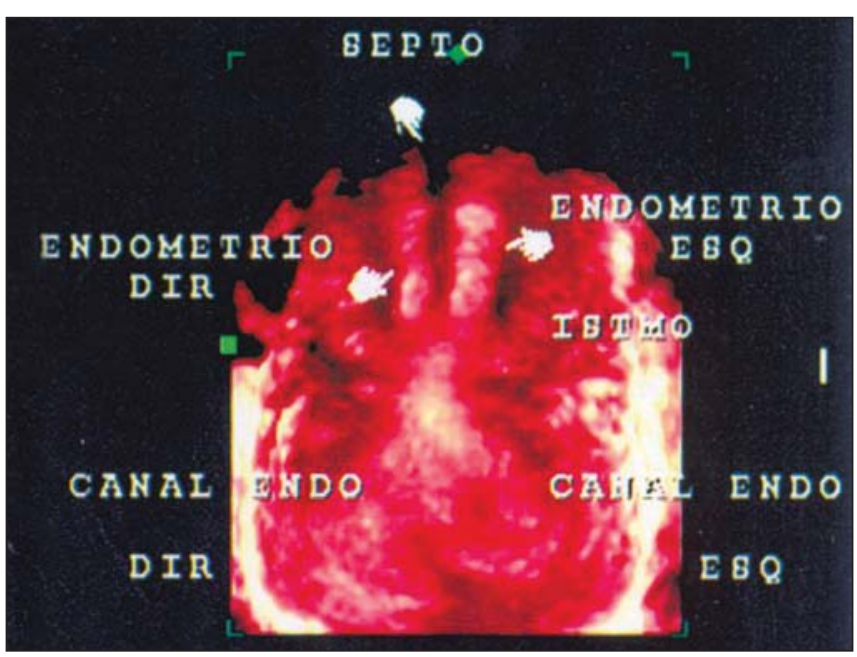

Figura 1 - Septo uterino e duplicação de canal endocervical. 


\section{Discussão}

Várias tentativas foram feitas durante anos para estabelecer uma classificação adequada das anomalias dos ductos müllerianos ${ }^{3,4,9,10}$. Estas classificações são compatíveis com as hipóteses sobre o desenvolvimento embriológico normal do sistema mülleriano assim como a formação das anomalias müllerianas. A teoria clássica, que sustenta que a fusão mülleriana ocorre de maneira unidirecional, no sentido caudal-cefálico, foi detalhada por Crosby e Hill, em $1962^{11}$.

Neste caso, descrevemos a duplicação cervical associada a septo vaginal e uterino com fundo uterino único, no qual houve gestação espontânea com boa evolução obstétrica. Esta rara anomalia foi primeiramente descrita em $1994^{2}$. Desde então, outros relatos foram feitos, alguns isolados e outros em séries, totalizando menos de 40 casos até o momento ${ }^{6,7,11,12}$. Após estas publicações, a clássica teoria do desenvolvimento unidirecional (caudal-cranial) dos ductos müllerianos foi questionada, sendo proposta uma teoria alternativa bidirecional, que sustenta que a fusão e a reabsorção iniciem no istmo e procedam, simultaneamente, em ambas as direções, cranial e caudal ${ }^{13}$. Concordando com esta teoria, McBean e Brumsted ${ }^{2}$ sugerem que o útero septado com duplicação cervical e septo vaginal longitudinal possa ser explicado pela falha na fusão de dois ductos müllerianos distais, com desenvolvimento normal do resto do útero, causando a duplicação cervical,

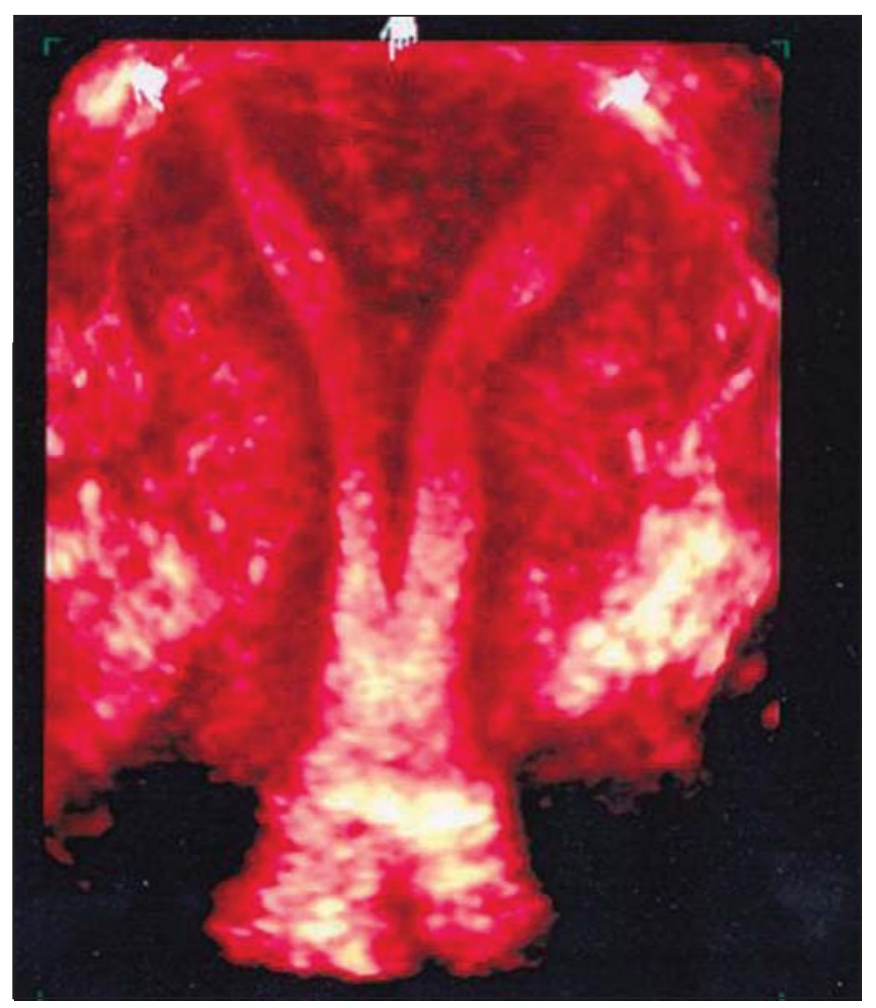

Figura 2 - Septo uterino e duplicação de canal endocervical. com a presença de um fundo uterino único. Já os septos uterino e vaginal são conseqüências da falha completa na reabsorção septal ou canalização. A teoria clássica da fusão unidirecional não consegue explicar adequadamente esta rara anomalia descrita.

Casos de septo uterino completo associado a septo vaginal podem ser facilmente confundidos com útero didelfo. Entretanto, o manejo destas duas anomalias müllerianas é diverso, sendo a correção cirúrgica usualmente necessária nos casos de septo uterino completo. No que tange aos propósitos diagnósticos dos relatos anteriores, Wai et al. ${ }^{14}$ enfatizaram a utilidade da histeroscopia laparoscopicamente assistida, enquanto que os estudos mais recentes propõem a ressonância nuclear magnética (RNM) para o diagnóstico definitivo e não invasivo desta rara anomalia ${ }^{7,11}$. O presente caso é interessante porque demonstra o valor da ecografia pélvica tridimensional como um método de imagem diagnóstico definitivo para avaliação da anatomia uterina e para o diagnóstico das anomalias müllerianas. A ultra-sonografia tridimensional é um exame menos invasivo e de menor custo que a histeroscopia/laparoscopia ou a RNM. A possibilidade de visualização da cavidade uterina e do miométrio com a ecografia tridimensional facilita o diagnóstico das anomalias uterinas, proporcionando a diferenciação entre septo uterino e útero bicorno, e permitindo ainda o planejamento cirúgico $^{15,16}$. No caso descrito, além da avaliação do corpo uterino (Figura 2), foi possível evidenciar a presença dos dois colos uterinos (Figura 1).

Do ponto de vista reprodutivo, o septo uterino é associado a abortamento, que classicamente ocorre entre oito e 16 semanas. Porém, a presença de septo uterino e duplicação cervical é uma malformação ainda pouco estudada e seu impacto sobre a fertilidade ainda não está elucidado. Patton et al. ${ }^{17}$ publicaram a maior série de casos desta anomalia, na qual avaliaram o diagnóstico, o manejo clínico e o resultado reprodutivo após o reparo cirúrgico desta malformação. Nesta série de 16 pacientes, todas eram sintomáticas, sendo que nove apresentavam queixas de perdas fetais e outras sete nuligrávidas tinham dispareunia. Em nenhum caso, o diagnóstico correto havia sido realizado antes do encaminhamento ao centro de referência. Todas as pacientes deste estudo realizaram tratamento cirúrgico, sendo que 12 engravidaram, totalizando 17 gestações. $O$ índice de perda fetal pré-operatório era de $81 \%$ e foi reduzido para $18 \%$. Estes dados são condizentes com a literatura no manejo dos septos uterinos, na qual os dados obtidos sugerem que a metroplastia histeroscópica está associada a um prognóstico favorável, e os índices de gestação são de $80 \%$ e as perdas de somente $15 \%$ após esse tipo de tratamento, quando consideradas somente as pacientes com abortos de repetição e infertilidade. 
No caso clínico apresentado, o tratamento cirúrgico não foi realizado devido ao fato de a paciente ter gestado durante o processo de investigação.

Em nosso caso, a paciente era assintomática, tanto do ponto de vista reprodutivo como clínico, e teve gestação espontânea sem intercorrências, com recémnascido saudável, a termo. Este achado poderia indicar que tratamentos intervencionistas estariam somente indicados nas mulheres com história de perdas fetais, trabalho de parto prematuro e outras intercorrências obstétricas que poderiam ser atribuídas a essa anomalia anatômica. Muitos especialistas em medicina reprodutiva concordam que a metroplastia está indicada nos casos de septo uterino associado a perdas fetais recorrentes; porém, há controvérsias no tratamento cirúrgico para pacientes assintomáticas ou nuligrávidas. Além disso, nos casos de malformações uterinas, a real avaliação do risco obstétrico é dificultada, porque muitas malformações não são diagnosticadas, especialmente nas pacientes assintomáticas ${ }^{18-21}$.

Alguns autores defendem que, frente a essa malformação, a correção cirúrgica teria um impacto importante sobre a fertilidade ${ }^{22}$, tendo índices de até $75 \%$ de gestação espontânea e diminuição da taxa de abortamentos. A realização de metroplastia profilática em pacientes com septo uterino sem história de abortamentos prévios ou complicações da gestação ainda está em discussão. O sucesso da implantação embrionária e o desenvolvimento fetal podem ocorrer sem metroplastia prévia, como no caso descrito por Weissman et al. ${ }^{23}$, que relatam um caso de gestação gemelar após fertilização in vitro sem intercorrências até 34 semanas, quando foi realizada cesariana com o nascimento de dois recém nascidos saudáveis.

Em casos de septo uterino completo e duplicação cervical, o correto diagnóstico é de fundamental importância, pois, uma vez que se opte pelo tratamento cirúrgico, a porção terminal cervical do septo (a junção medial fusionada do colo duplo) deve ser preservada durante a metroplastia, evitando sangramento cirúrgico e incompetência istmo-cervical no futuro.

Este é um dos raros casos descritos desta malformação, sem intervenção cirúrgica prévia, no qual ocorreu gestação espontânea que evoluiu sem intercorrências. Essa malformação, na maioria das vezes, tem o diagnóstico tardio, geralmente na idade reprodutiva, devido ao fato de não causar problemas durante a puberdade. Como ainda não existe consenso quanto ao impacto desta anomalia sobre a fertilidade, estas pacientes devem ser seguidas no intuito de se estabelecer o melhor plano terapêutico a ser seguido, especialmente naquelas assintomáticas. $\mathrm{O}$ crescente número de casos descritos desta anomalia, desde o primeiro relato em 1994, deve ser resultado do aumento de acesso e acurácia dos métodos de imagem, como a RNM e a ecografia tridimensional. A ultra-sonografia tridimensional, exame não invasivo e de baixo custo, é uma opção diagnóstica que deve ser considerada no arsenal semiótico das malformações müllerianas.

\section{Referências}

1. Acién $P$, Acién $M$, Sánchez-Ferrer M. Complex malformations of the female genital tract. New types and revision of classification. Hum Reprod. 2004; 19(10):2377-84.

2. McBean JH, Brumsted JR. Septate uterus with cervical duplication: a rare malformation. Fertil Steril 1994;62(3):415-7.

3. Buttram VC Jr, Gibbons WE. Müllerian anomalies: a proposed classification (an analysis of 144 cases). Fertil Steril. 1979;32(1):40-6.

4. The American Fertility Society classifications of adnexal adhesions, distal tubal occlusion tubal occlusion secondary to tubal ligation, tubal pregnancies, müllerian anomalies and intrauterine adhesions. Fertil Steril. 1988;49(6):944-55.

5. Martinez-Frias ML, Bermejo Sánchez E, Rodríguez-Pinilla $E$, Martinez Santana S, Paisan Grisolia L, Egues Jimeno J, et al. Epidemiological aspects of children of women with bicornuate uterus. An Esp Pediatr. 1998;48(2):159-62.

6. Saygili-Yilmaz ES, Erman-Akar M, Bayar D, Yuksel B, Yilmaz $Z$. Septate uterus with a double cervix and longitudinal vaginal septum. J Reprod Med. 2004;49(10):833-6.
7. Chang AS, Siegel CL, Moley KH, Ratts VS, Odem RR. Septate uterus with cervical duplication and longitudinal vaginal septum: a report of five new cases. Fertil Steril. 2004;81(4):1133-6.

8. Badalotti M, Arent A, Monteggia V, Machado J, Petracco R, Petracco A. Septate uterus, cervical duplication and vaginal septum: a report of an uncommon malformation. Rev Colomb Obstet Ginecol. 2005;56(3):244-8.

9. Shuiqing $M$, Xuming $B$, Jinghe L. Pregnancy and its outcome in women with malformed uterus. Chin Med Sci J. 2002; 17(4):242-5.

10. Balasch J, Moreno E, Martinez-Roman S, Molini JL, Torne A, Sanchez-Martin F, et al. Septate uterus with cervical duplication and longitudinal vaginal septum: a report of three new cases. Eur J Obstet Gynecol Reprod Biol. 1996;65(2):241-3.

11. Crosby WM, Hill EC. Embriology of the müllerian duct system: review of present-day theory. Obstet Gynecol. 1962;20(4):507-15.

12. Hundley AF, Fielding JR, Hoyte L. Double cervix and vagina with septate uterus: an uncommom müllerian malformation. Obstet Gynecol. 2001;98(5 Pt 2):982-5. 
13. Muller P, Musset R, Netter A, Solal R, Vinourd JC, Gillet JY. Etat di haut appareil urinaire chez les porteuses de malformations uterines:stude de 133 observations. Presse Med. 1967;75(26):1331-6.

14. Wai CY, Zekam N, Sanz LE. Septate uterus with double cervix and longitudinal vaginal septum. A case report. J Reprod Med. 2001;46(6):613-7.

15. Kupesic S, Kurjak A. Diagnosis and treatment outcome of the septate uterus. Croat Med J. 1998;39(2):185-90.

16. Wu MH, Hsu CC, Huang KE. Detection of congenital müllerian duct anomalies using three-dimensional ultrasound. J Clin Ultrasound. 1997;25(9):487-92.

17. Patton PE, Novy M, Lee DM, Hickok LR. The diagnosis and reproductive outcome after surgical treatment of the complete septate uterus, duplicated cervix and vaginal septum. Am J Obstet Gynecol. 2004;190(6): 1669-75.

18. Narlawar RS, Chavhan GB, Bhatgadde VL, Shah JR. Twin gestation in one horn of bicornuate uterus. J Clin Ultrassound. 2003;31(3):167-9.
19. Ma S, Bian X, Lang J. Pregnancy and its outcomes in women with malformed uterus. Zhonghua Yi Xue Za Zhi. $2001 ; 81(7): 415-7$.

20. Brun JL, Lemoine P. Uterine malformations. Diagnosis, prognosis and treatment. Presse Med. 1995;24(35):1658-62.

21. Bongain A, Constantopoulos P, Castillon JM, Ibghi W, Isnard V, Gillet JY. Simultaneous pregnancy in each cavity of bicornuate bicervical uterus with a double vagina. Rev Fr Gynecol Obstet. 1994;89(1):32-5.

22. Pace S, Cipriano L, Pace G, Catania R, Montanino G. Septate uterus: reproductive outcome after hysteroscopic metroplasty. Clin Exp Obstet Gynecol. 2006;33(2): 1 10-2.

23. Weissman A, Eldar I, Malinger G, Sadan O, Glezerman $M$, Levran D. Successful twin pregnancy in a patient with complete uterine septum corrected during cesarean section. Fertil Steril. 2006;85(2):494.e 1-494.e4. 